Supplement of Atmos. Chem. Phys., 16, 3577-3594, 2016

http://www.atmos-chem-phys.net/16/3577/2016/

doi:10.5194/acp-16-3577-2016-supplement

(C) Author(s) 2016. CC Attribution 3.0 License.

(c) (i)
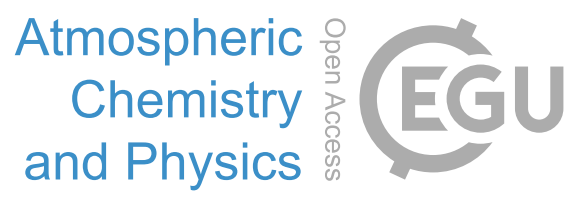

Supplement of

\title{
The importance of vehicle emissions as a source of atmospheric ammonia in the megacity of Shanghai
}

\author{
Y. H. Chang et al. \\ Correspondence to:
}

The copyright of individual parts of the supplement might differ from the CC-BY 3.0 licence. 


\section{This file includes:}

\section{Figures}

Fig. S1 Comparison of results obtained with the Ogawa $\mathrm{NH}_{3}$ PSDs and an active monitor (MARGA) at Pudong supersite from May 2014 to June 2015.

Fig. S2 RH/T dependence of $\mathrm{NH}_{3}$ concentration in spring (a), summer (b), fall (c), and winter (d) at Pudong supersite during April 3, 2014-April 2, 2015.

Fig. S3 WS/WD dependence of $\mathrm{NH}_{3}$ concentrations in spring (a), summer (b), fall (c), and winter (d) at Pudong supersite during April 3, 2014-April 2, 2015.

Fig. S4 WRF model domain and location of the NCDC surface meteorological network.

\section{Table}

Table S1. Descriptive statistics of the $\mathrm{NH}_{3}$ concentration $\left(\mu \mathrm{g} \mathrm{m}^{-3}\right)$ at different sampling sites in and out of the Handan tunnel during the spring campaign in 2014. See Figure 7a and the method section for the information on the sampling points.emission sources

Table S2. Evaluation of WRF simulations and NCDC measured results of surface wind speed, temperature, and humidity. 


\section{Figures}

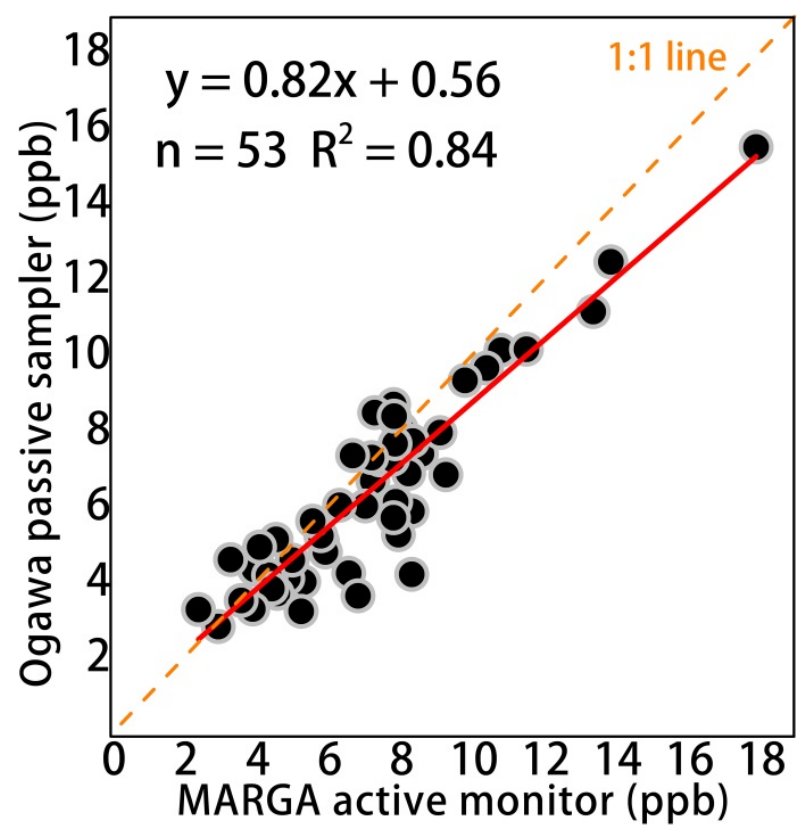

Figure S1. Comparison of results obtained with the Ogawa $\mathrm{NH}_{3} \mathrm{PSDs}$ and an active monitor (MARGA) at Pudong supersite from May 2014 to June 2015. 

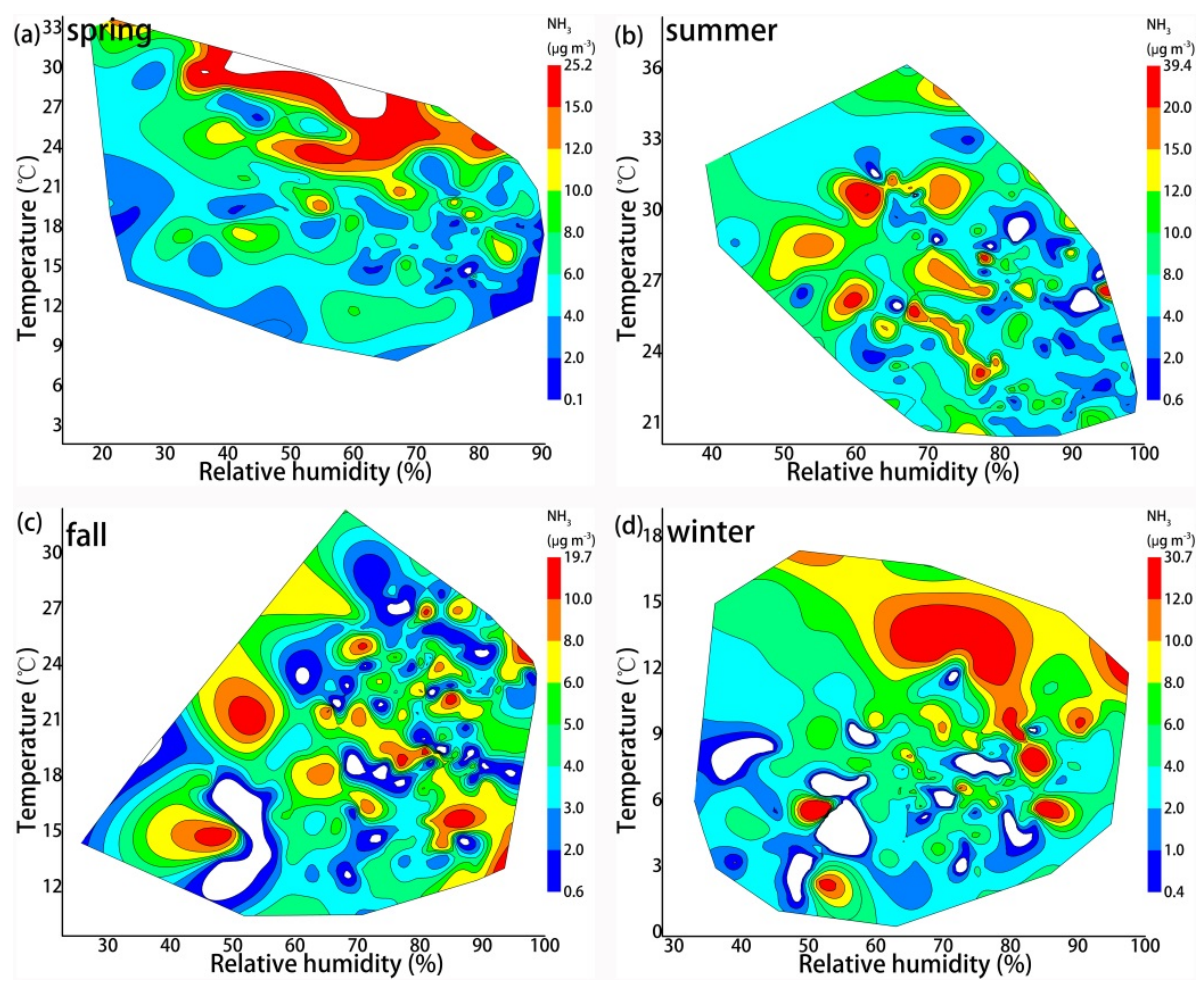

Figure S2. $\mathrm{RH} / \mathrm{T}$ dependence of $\mathrm{NH}_{3}$ concentration in spring (a), summer (b), fall (c), and winter (d) at Pudong supersite during April 2, 2014-April 3, 2015. 

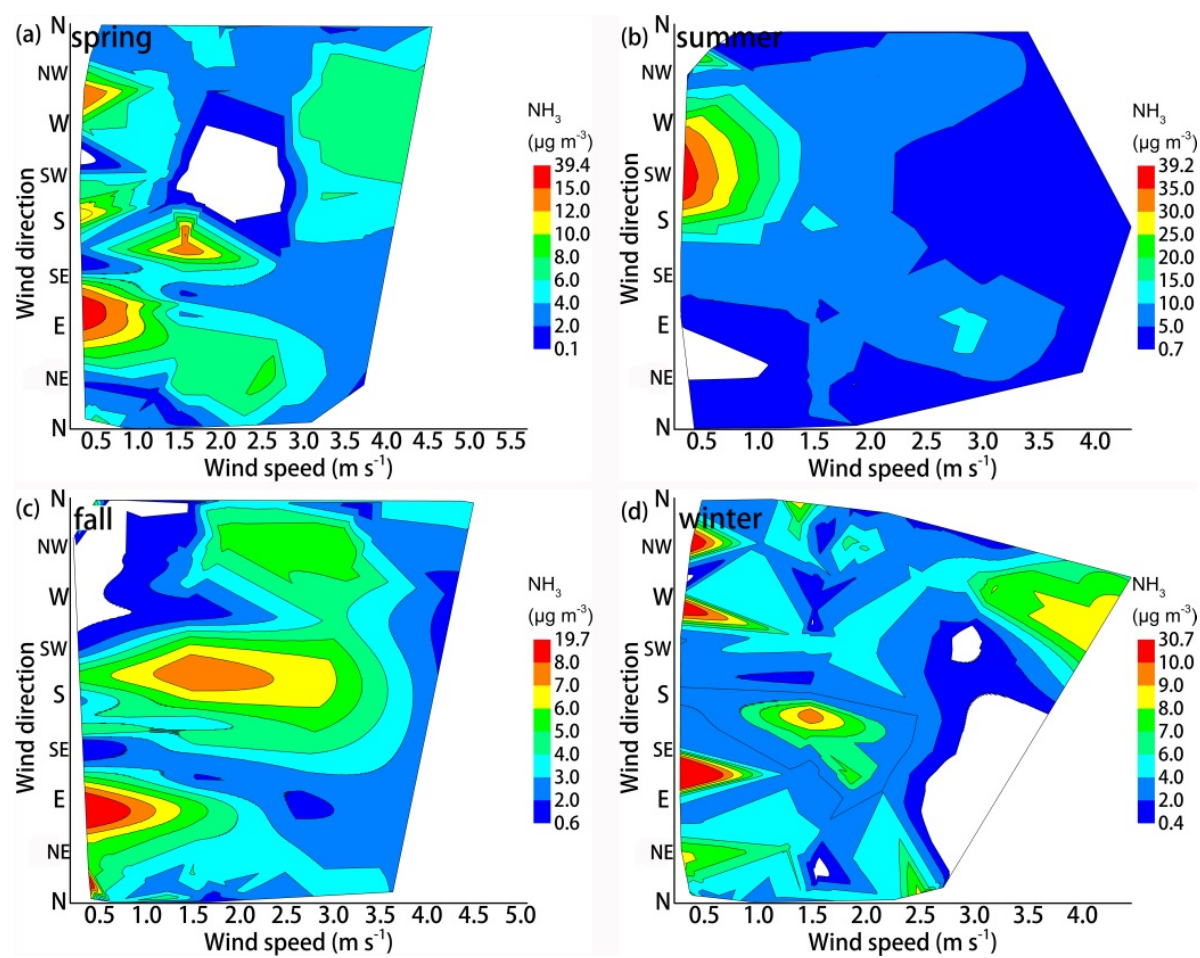

Figure S3. WS/WD dependence of $\mathrm{NH}_{3}$ concentrations in spring (a), summer (b), fall (c), and winter (d) at Pudong supersite during April 2, 2014-April 3, 2015. 


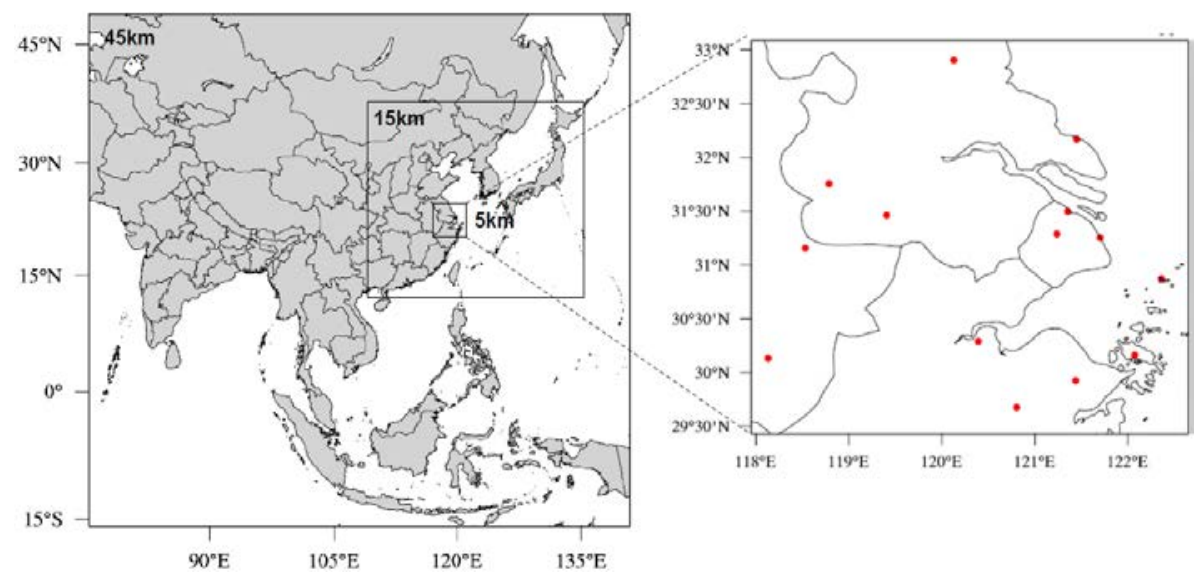

Fig. S4 WRF model domain and location of the NCDC surface meteorological network. 


\section{Table}

Table S1. Descriptive statistics of the $\mathrm{NH}_{3}$ concentration $\left(\mu \mathrm{g} \mathrm{m}^{-3}\right)$ at different sampling sites in and out of the Handan Tunnel during the spring campaign in 2014.

See Figure 7a and the method section for the information on the sampling points.

\begin{tabular}{clcccccc}
\hline & Sampling point & $\mathrm{N}$ & Mean & SD & Minimum & Median & Maximum \\
\hline \multirow{2}{*}{ Handan } & T-a & 6 & 12.6 & 3.3 & 8.8 & 12.3 & 18.1 \\
Tunnel & T-b & 6 & 29.2 & 6.6 & 20.1 & 28.6 & 38.7 \\
& T-c & 6 & 31.5 & 5.9 & 21.4 & 33.3 & 37.6 \\
& T-d & 19 & 64.9 & 11.5 & 47.0 & 65.4 & 82.9 \\
\hline \multirow{2}{*}{ Open } & $\mathrm{O}_{0 \mathrm{~m}}$ & 19 & 11.7 & 4.2 & 7.5 & 10.7 & 25.0 \\
& $\mathrm{O}_{20 \mathrm{~m}}$ & 19 & 6.5 & 2.8 & 2.8 & 5.8 & 13.2 \\
environment & $\mathrm{O}_{150 \mathrm{~m}}$ & 19 & 5.9 & 2.5 & 2.1 & 5.1 & 10.7 \\
& $\mathrm{O}_{310 \mathrm{~m}}$ & 19 & 5.6 & 2.5 & 1.9 & 4.9 & 10.1 \\
\hline
\end{tabular}


Table S2. Evaluation of WRF simulations and NCDC measured results of surface wind speed, temperature, and humidity.

\begin{tabular}{|c|c|c|c|c|c|c|c|c|c|c|c|c|c|}
\hline & & JAN & FEB & MAR & APR & MAY & JUN & JUL & AUG & SEP & OCT & NOV & DEC \\
\hline Wind Speed $(\mathrm{m} / \mathrm{s})$ & Mean Obs & 2.56 & 2.87 & 2.98 & 3.18 & 3.65 & 3.22 & 3.86 & 3.37 & 3.34 & 2.81 & 2.51 & 2.43 \\
\hline Benchmark & Mean Sim & 3.08 & 3.38 & 2.88 & 3.23 & 3.92 & 2.94 & 3.5 & 3.45 & 3.38 & 2.94 & 2.84 & 2.62 \\
\hline$\leq \pm 0.5$ & Mean Bias & 0.52 & 0.51 & -0.1 & 0.05 & 0.27 & -0.28 & -0.36 & 0.08 & 0.04 & 0.13 & 0.33 & 0.19 \\
\hline \multirow[t]{2}{*}{$\leq 2$} & Gross Error & 1.3 & 1.29 & 1.19 & 1.36 & 1.53 & 1.16 & 1.22 & 1.21 & 1.14 & 1.11 & 1.33 & 1.22 \\
\hline & RMSE & 1.72 & 1.72 & 1.65 & 1.79 & 1.94 & 1.51 & 1.59 & 1.54 & 1.44 & 1.4 & 1.74 & 1.6 \\
\hline$\geq 0.6$ & IOA & 0.77 & 0.77 & 0.81 & 0.78 & 0.66 & 0.73 & 0.74 & 0.72 & 0.79 & 0.74 & 0.79 & 0.78 \\
\hline Temperature (K) & Mean Obs & 272.95 & 278.54 & 281.18 & 287.25 & 293.62 & 298.5 & 301.93 & 302.64 & 296.17 & 294.34 & 281.42 & 274.53 \\
\hline Benchmark & Mean Sim & 273.63 & 278.3 & 281.16 & 287.09 & 293.8 & 298.84 & 301.89 & 302.27 & 296.57 & 294.68 & 282.45 & 275.72 \\
\hline$\leq \pm 0.5$ & Mean Bias & 0.68 & -0.24 & -0.02 & -0.16 & 0.18 & 0.34 & -0.04 & -0.37 & 0.4 & 0.34 & 1.03 & 1.19 \\
\hline \multirow[t]{2}{*}{$\leq 2$} & Gross Error & 1.29 & 1.32 & 1.41 & 1.47 & 1.68 & 1.71 & 1.39 & 1.26 & 1.28 & 1.23 & 1.51 & 1.69 \\
\hline & RMSE & 1.61 & 1.75 & 1.83 & 1.89 & 2.09 & 2.19 & 1.8 & 1.61 & 1.59 & 1.57 & 1.86 & 2.06 \\
\hline$\geq 0.8$ & IOA & 0.98 & 0.97 & 0.96 & 0.94 & 0.86 & 0.87 & 0.85 & 0.9 & 0.89 & 0.91 & 0.98 & 0.97 \\
\hline Humidity (g/kg) & Mean Obs & 3.61 & 4.15 & 4.84 & 6.93 & 11.18 & 16.35 & 20.4 & 19.98 & 13.97 & 12.22 & 5.72 & 3.72 \\
\hline Benchmark & Mean Sim & 4.26 & 3.67 & 4.9 & 7.36 & 10.2 & 14.92 & 19.28 & 19.21 & 13.24 & 11.36 & 6.68 & 4.36 \\
\hline$\leq \pm 1$ & Mean Bias & 0.65 & -0.48 & 0.06 & 0.43 & -0.98 & -1.43 & -1.12 & -0.77 & -0.73 & -0.86 & 0.96 & 0.64 \\
\hline \multirow[t]{2}{*}{$\leq 2$} & Gross Error & 0.51 & 0.92 & 0.92 & 1.45 & 1.54 & 1.7 & 1.06 & 1.02 & 0.98 & 1.34 & 1.15 & 0.72 \\
\hline & RMSE & 0.63 & 1.13 & 1.13 & 1.76 & 1.93 & 2.41 & 1.56 & 2.14 & 1.43 & 1.7 & 1.38 & 0.87 \\
\hline$\geq 0.6$ & IOA & 0.86 & 0.78 & 0.78 & 0.73 & 0.59 & 0.6 & 0.71 & 0.72 & 0.69 & 0.58 & 0.87 & 0.87 \\
\hline
\end{tabular}

$$
\text { Mean Bias }=\frac{1}{N} \sum_{i=1}^{N} C_{m}-C_{o}, \mathrm{GE}=\frac{1}{N} \sum_{i=1}^{N}\left|C_{m}-C_{o}\right|, \mathrm{RMSE}=\sqrt{\frac{1}{N} \sum_{i=1}^{N}\left(C_{m}-C_{o}\right)^{2}}, \mathrm{IOA}=1-\frac{\sum_{i=1}^{N}\left(C_{m}-C_{o}\right)^{2}}{\sum_{i=1}^{N}\left(\left|C_{m}-\overline{C_{o}}\right|+\left|C_{o}-\overline{C_{o}}\right|\right)^{2}}
$$

